PROCEEDINGS OF THE

AMERICAN MATHEMATICAL SOCIETY

Volume 133, Number 5, Pages 1547-1556

S 0002-9939(04)07633-6

Article electronically published on November 19, 2004

\title{
METRICALLY GENERATED THEORIES
}

\author{
E. COLEBUNDERS AND R. LOWEN
}

(Communicated by Alan Dow)

\begin{abstract}
Many examples are known of natural functors $K$ describing the transition from categories $\mathcal{C}$ of generalized metric spaces to the "metrizable" objects in some given topological construct $\mathcal{X}$. If $K$ preserves initial morphisms and if $K(\mathcal{C})$ is initially dense in $\mathcal{X}$, then we say that $\mathcal{X}$ is $\mathcal{C}$-metrically generated. Our main theorem proves that $\mathcal{X}$ is $\mathcal{C}$-metrically generated if and only if $\mathcal{X}$ can be isomorphically described as a concretely coreflective subconstruct of a model category with objects sets structured by collections of generalized metrics in $\mathcal{C}$ and natural morphisms. This theorem allows for a unifying treatment of many well-known and varied theories. Moreover, via suitable comparison functors, the various relationships between these theories are studied.
\end{abstract}

\section{INTRODUCTION}

Metrics (or any of their various generalizations) lie at the basis of many topological and analytical theories. Natural functors describe the transition from (generalized) metric spaces to objects in a given category $\mathcal{X}$. With a (generalized) metric $d$ one can associate e.g. a topology $\mathcal{T}_{d}$, a uniformity $\mathcal{U}_{d}$, an approach structure $\mathcal{A}_{d}$, a bornological structure $\mathcal{B}_{d}$ or a $\sigma$-algebra $\sigma\left(\mathcal{T}_{d}\right)$. In each of these examples, a natural functor $K$ from a category of (generalized) metric spaces to the category $\mathcal{X}$ is given. Some of these examples give rise to intuitive notions of what one could call a "metrically generated theory". For instance (1) every uniform space, as well as every approach space, is a subspace of a product of "metrizable" spaces. It is also known that (2) for both these categories the objects can be isomorphically described by means of sets structured by collections of "metrics" 7], [10].

In this paper we investigate the relationship between the two points of view (1) and (2). In our main theorem 4.1, for specific constructs $\mathcal{C}$ of (generalized) metric spaces, we give necessary and sufficient conditions on a functor $K: \mathcal{C} \rightarrow \mathcal{X}$ in order to characterize when $\mathcal{X}$ can be isomorphically described as a full concretely coreflective subconstruct of a model category $\mathbf{M}^{\mathcal{C}}$ with objects sets structured by collections of (generalized) $\mathcal{C}$-metrics. Topological constructs $\mathcal{X}$ for which there exists a functor $K: \mathcal{C} \rightarrow \mathcal{X}$ satisfying these necessary and sufficient conditions are called $\mathcal{C}$-metrically generated. So as it turns out, the settings $\mathbf{M}^{\mathcal{C}}$ for various

Received by the editors September 22, 2003 and, in revised form, January 5, 2004.

2000 Mathematics Subject Classification. Primary 54B30, 18B99, 18E20.

Key words and phrases. Topological construct, topological space, metric space, uniform space, approach space, bornological space, measurable space. 
$\mathcal{C}$ are of sufficient generality to provide a unifying treatment of the $\mathcal{C}$-metrically generated theories, in particular allowing for one common characterization of a plethora of various types of morphisms as e.g. continuous, uniformly continuous, proximal, bounded and measurable maps. Once the theories are classified using various $\mathcal{C}$ and different expanders as introduced in Definition 3.2, in Theorem 3.5 we describe suitable comparison functors to study the relationship between them. In view of their tight connection with "metrizable" objects, it turns out that the constructs $\mathbf{M}^{\mathcal{C}}$ and their subconstructs provide a very advantageous setting for the development of compactness and/or completeness theory, which is the subject of a subsequent paper. The classification is exemplified in the sections on examples and counterexamples. Of course well-known examples are easily captured: Top, Unif and Ap are metrically generated. But the characterization also allows us to produce less evident examples such as measurable spaces or bornological spaces derived from an $l^{\infty}$ structure as described in [6]. The same characterization also allows us to prove e.g. that although the construct Bor of all bornological spaces has a natural functor assigning a bornology to a metric, this category, nevertheless, is not metrically generated.

\section{Metrically Generated CONSTRUCts}

For notational and terminological simplicity and in order not to deviate too much from standard practice, we will call a function $d: X \times X \rightarrow[0, \infty]$ a quasipre-metric if it is zero on the diagonal, we will drop "pre" if $d$ satisfies the triangle inequality and we will drop "quasi" if $d$ is symmetric. Note in particular that we do not suppose a metric to be either real-valued or separated. We refer to [1] for all categorical concepts and terminology. Met stands for the construct of quasipre-metric spaces and contractions. A map $f:(X, d) \rightarrow\left(X^{\prime}, d^{\prime}\right)$ is a contraction if $d^{\prime} \circ f \times f \leq d$. If $\mathcal{A}$ is a construct and $X$ a set, then $\mathcal{A}(X)$ stands for the fiber of $\mathcal{A}$-structures on $X$.

2.1. Definition. A full and isomorphism-closed concrete subconstruct of Met which is closed for initial morphisms and which contains all Met-indiscrete spaces will be called a base category. Given a base category $\mathcal{C}$, a topological construct $\mathcal{X}$ will be called $\mathcal{C}$-metrically generated if there exists a concrete functor $K: \mathcal{C} \rightarrow \mathcal{X}$ satisfying the following two properties: (I) $K$ preserves initial morphisms, (D) $K(\mathcal{C})$ is initially dense in $\mathcal{X}$. We will then also say that $\mathcal{X}$ is metrically generated by $K$.

In the following two propositions we give some basic results concerning the properties (I) and (D). Note that these results hold for analogous functors and categories, and it suffices to replace $d \circ f \times f$ by the initial structure via $f$. If $\mathcal{C}$ is a base category and $(X, d)$ is an object of $\mathcal{C}$, we will call $d$ a $\mathcal{C}$-metric. In the sequel $\mathcal{C}$ will always denote a base category.

2.2. Proposition. (1) $K$ satisfies (I) if and only if for any $\mathcal{X}$-morphism $f: \underline{X} \rightarrow \underline{X}^{\prime}$ and for any $\mathcal{C}$-metric $q$ on $X^{\prime}$, if $K\left(X^{\prime}, q\right) \leq \underline{X^{\prime}}$, then $K(X, q \circ f \times f) \leq \underline{X}$.

(2) In the presence of $(I), K$ satisfies $(D)$ if and only if for any $\mathcal{X}$-object $\underline{X}$ there exists a collection of $\mathcal{C}$-metrics $\mathcal{P}$ such that $\underline{X}=\sup _{p \in \mathcal{P}} K(X, p)$. 
Proof. 1. Suppose (I) is fulfilled, let $f: \underline{X} \rightarrow \underline{X}^{\prime}$ be a morphism and suppose $K\left(X^{\prime}, p\right) \leq \underline{X}^{\prime}$. If we consider the following diagram,

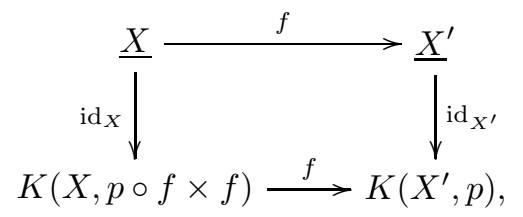

then since the bottom $f$ is initial, clearly $\operatorname{id}_{X}$ is a morphism.

Conversely, consider the diagram

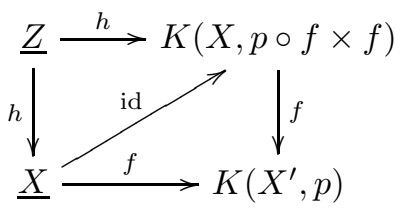

where $h: \underline{Z} \rightarrow K(X, p \circ f \times f)$ is a function such that $f \circ h$ is a morphism, and where $h: \underline{Z} \rightarrow \underline{X}$ is final. Then it follows that the bottom $f$ is a morphism. The condition stated in the proposition now implies that id is a morphism and hence that also $h: \underline{Z} \rightarrow K(X, p \circ f \times f)$ is a morphism.

2. Let $\underline{X}$ be an object in $\mathcal{X}$, and let $\left(f_{i}: \underline{X} \rightarrow K\left(X_{i}, p_{i}\right)\right)_{i \in I}$ be an initial source in $\mathcal{X}$. Since $f_{i}: \underline{X} \rightarrow K\left(X_{i}, p_{i}\right)$ is a morphism in $\mathcal{X}$, it follows from (I) that $K\left(X, p_{i} \circ f_{i} \times f_{i}\right) \leq \underline{X}$. Then consider the composition of the sources in $\mathcal{X}$ :

$$
\underline{X} \stackrel{\text { id }}{\longrightarrow} K\left(X, p_{i} \circ f_{i} \times f_{i}\right) \stackrel{f_{i}}{\longrightarrow} K\left(X_{i}, p_{i}\right) .
$$

Since this composition is initial, it follows that the first source is initial and hence $\underline{X}=\sup _{i \in I} K\left(X, p_{i} \circ f_{i} \times f_{i}\right)$.

2.3. Proposition. A functor $K: \mathcal{C} \rightarrow \mathcal{X}$, satisfying (D) preserves indiscrete objects.

Proof. Let $\left(X, d_{0}\right)$ be indiscrete. For $\underline{X}$ indiscrete in $\mathcal{X}$, by (D), we have that there is a set of $\mathcal{C}$-metrics $\mathcal{P}$ such that $\underline{X}=\sup _{p \in \mathcal{P}} K(X, p)$. Take $p \in \mathcal{P}$; then $K(X, p)$ is indiscrete and $d_{0} \leq p$. Hence $K\left(X, d_{0}\right) \leq K(X, p)$, and it follows that $K\left(X, d_{0}\right)$ is indiscrete.

\section{METERED SPACES AND EXPANDERS}

We now construct a model category for $\mathcal{C}$-metrically generated constructs. First, we need some preliminary concepts and results. A downset in $\operatorname{Met}(X)$ is a nonempty subset $\mathcal{S} \subset \boldsymbol{M e t}(X)$ such that if $d \in \mathcal{S}, e \in \operatorname{Met}(X)$ and $e \leq d$, then $e \in \mathcal{S}$. For $\mathcal{B} \subset \operatorname{Met}(X), \mathcal{B} \downarrow:=\{e \in \operatorname{Met}(X) \mid \exists d \in \mathcal{B}: e \leq d\}$.

3.1. Definition. We define $\mathbf{M}$ to be the construct with objects pairs $(X, \mathcal{M})$ where $X$ is a set and $\mathcal{M}$ is a downset in $\operatorname{Met}(X) . \mathcal{M}$ is called a meter (on $X)$ and $(X, \mathcal{M})$ a metered space. If $(X, \mathcal{M})$ and $\left(X^{\prime}, \mathcal{M}^{\prime}\right)$ are metered spaces and $f:(X, \mathcal{M}) \rightarrow$ $\left(X^{\prime}, \mathcal{M}^{\prime}\right)$, then we say that $f$ is a contraction if

$$
\forall d^{\prime} \in \mathcal{M}^{\prime}: d^{\prime} \circ f \times f \in \mathcal{M} \text {. }
$$


It is easily verified that $\mathbf{M}$ is a topological construct. Given a structured source $\left(f_{j}: X \rightarrow\left(X_{j}, \mathcal{M}_{j}\right)\right)_{j \in J}$, the initial structure on $X$ is the meter

$$
\left\{d \circ f_{j} \times f_{j} \mid j \in J, d \in \mathcal{M}_{j}\right\} \downarrow .
$$

Analogously, given a structured $\operatorname{sink}\left(f_{j}:\left(X_{j}, \mathcal{M}_{j}\right) \rightarrow X\right)_{j \in J}$, the final structure on $X$ is the meter

$$
\left\{d \in \operatorname{Met}(X) \mid \forall j \in J: d \circ f_{j} \times f_{j} \in \mathcal{M}_{j}\right\} .
$$

Given a meter $\mathcal{M}$ we will say that $\mathcal{B} \subset \mathcal{M}$ is a basis for $\mathcal{M}$ if $\mathcal{B} \downarrow=\mathcal{M}$.

$\mathbf{M}^{\mathcal{C}}$ is the full subconstruct with objects, the meters of which have a basis consisting of $\mathcal{C}$-metrics. We will call such a meter a $\mathcal{C}$-meter. It follows from the fact that $\mathcal{C}$ is closed for initial morphisms that $\mathbf{M}^{\mathcal{C}}$ is a concretely reflective subconstruct of $\mathbf{M}$, and thus a topological construct in its own right. In order to easily deal with concretely coreflective subconstructs of $\mathbf{M}^{\mathcal{C}}$, we formalize a notion which belongs to the folklore of the subject.

We formulate 3.2 and 3.3 in the context of the paper, but an analogous definition and characterization are valid in any topological construct.

3.2. Definition. We call $\xi$ an expander on $\mathbf{M}^{\mathcal{C}}$ if for any $X$ and any meter $\mathcal{M} \in$ $\mathbf{M}^{\mathcal{C}}(X), \xi$ provides us with a meter $\xi(\mathcal{M}) \in \mathbf{M}^{\mathcal{C}}(X)$ in such a way that the following properties are fulfilled:

E1. $\mathcal{M} \subset \xi(\mathcal{M})$,

E2. $\mathcal{M} \subset \mathcal{N} \Rightarrow \xi(\mathcal{M}) \subset \xi(\mathcal{N})$,

E3. $\xi(\xi(\mathcal{M}))=\xi(\mathcal{M})$,

E4. if $f: Y \rightarrow X$ and $\mathcal{M} \in \mathbf{M}^{\mathcal{C}}(X)$, then $\xi(\mathcal{M}) \circ f \times f \subset \xi(\mathcal{M} \circ f \times f \downarrow)$.

Here, for any meter $\mathcal{M}$, we have put $\mathcal{M} \circ f \times f:=\{d \circ f \times f \mid d \in \mathcal{M}\}$.

Given an expander $\xi$ on $\mathbf{M}^{\mathcal{C}}$, we define $\mathbf{M}_{\xi}^{\mathcal{C}}$ as the full subconstruct of $\mathbf{M}^{\mathcal{C}}$ with objects those metered spaces $(X, \mathcal{M})$ for which $\xi(\mathcal{M})=\mathcal{M}$.

3.3. Proposition. For any expander $\xi$ on $\mathbf{M}^{\mathcal{C}}, \mathbf{M}_{\xi}^{\mathcal{C}}$ is a concretely coreflective subconstruct of $\mathbf{M}^{\mathcal{C}}$ and, conversely, any concretely coreflective subconstruct of $\mathbf{M}^{\mathcal{C}}$ is so obtained.

For any meter $\mathcal{D}$ we put $[\mathcal{D}]^{\mathcal{C}}:=\{d \in \mathcal{D} \mid d$ a $\mathcal{C}$-metric $\}$.

3.4. Proposition. If $\mathcal{C}$ and $\mathcal{C}^{\prime}$ are base categories such that $\mathcal{C}^{\prime} \hookrightarrow \mathcal{C}$, and $\xi$ is an expander on $\mathbf{M}^{\mathcal{C}}$, then

$$
\xi^{\prime}(\mathcal{D}):=[\xi(\mathcal{D})]^{\mathcal{C}^{\prime}} \downarrow \quad \forall \mathcal{C}^{\prime} \text {-meter } \mathcal{D}
$$

defines an expander on $\mathbf{M}^{\mathcal{C}^{\prime}}$.

Proof. By straightforward verification.

We call $\xi^{\prime}$ the $\mathcal{C}^{\prime}$-modification of $\xi$. We define $\mathbf{M}^{\left(\xi, \mathcal{C}^{\prime}\right)}$ as the full subconstruct of $\mathbf{M}_{\xi}^{\mathcal{C}}$ with objects $(X, \xi(\mathcal{D}))$ where $\mathcal{D}$ is a $\mathcal{C}^{\prime}$-meter. Note that $\mathbf{M}^{\mathcal{C}^{\prime}}=\mathbf{M}^{\left(\xi, \mathcal{C}^{\prime}\right)}$ where $\xi(\mathcal{D})=\mathcal{D}$ for all $\mathcal{C}$-meters $\mathcal{D}$.

3.5. Theorem. If $\mathcal{C}$ and $\mathcal{C}^{\prime}$ are base categories such that $\mathcal{C}^{\prime} \hookrightarrow \mathcal{C}$, and $\xi$ is an expander on $\mathbf{M}^{\mathcal{C}}$, then:

(1) $\mathbf{M}_{\xi^{\prime}}^{\mathcal{C}^{\prime}}$ and $\mathbf{M}^{\left(\xi, \mathcal{C}^{\prime}\right)}$ are concretely isomorphic;

(2) $\mathbf{M}^{\left(\xi, \mathcal{C}^{\prime}\right)}$ is concretely reflective in $\mathbf{M}_{\xi}^{\mathcal{C}}$ and $\mathbf{M}^{\mathcal{C}^{\prime}}$ is concretely reflective in $\mathbf{M}^{\mathcal{C}}$ 
(3) $\mathbf{M}_{\xi^{\prime}}^{\mathcal{C}^{\prime}}$ is concretely coreflective in $\mathbf{M}^{\mathcal{C}^{\prime}}$ and $\mathbf{M}_{\xi}^{\mathcal{C}}$ is concretely coreflective in $\mathbf{M}^{\mathcal{C}}$.

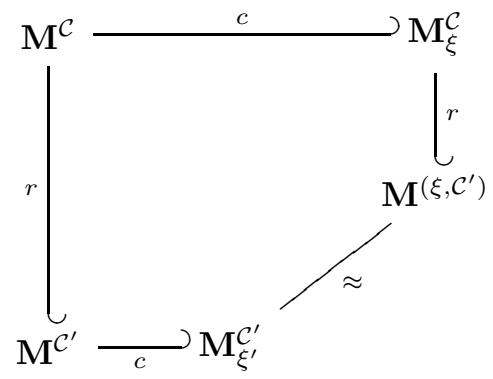

Proof. 1. Consider the concrete functor $G: \mathbf{M}^{\left(\xi, \mathcal{C}^{\prime}\right)} \rightarrow \mathbf{M}_{\xi^{\prime}}^{\mathcal{C}^{\prime}}$ that is defined by $G(X, \xi(\mathcal{Q} \downarrow)):=\left(X,[\xi(\mathcal{Q} \downarrow)]^{\mathcal{C}^{\prime}} \downarrow\right)$ where $\mathcal{Q}$ is a collection of $\mathcal{C}^{\prime}$-metrics. Remark that $\xi\left([\xi(\mathcal{Q} \downarrow)]^{\mathcal{C}^{\prime}} \downarrow\right)=\xi(\mathcal{Q} \downarrow)$, and hence $\xi^{\prime}\left([\xi(\mathcal{Q} \downarrow)]^{\mathcal{C}^{\prime}} \downarrow\right)=[\xi(\mathcal{Q} \downarrow)]^{\mathcal{C}^{\prime}} \downarrow$. Consequently the codomain of $G$ is indeed $\mathbf{M}_{\xi^{\prime}}^{\mathcal{C}^{\prime}}$. Next consider the concrete functor $F: \mathbf{M}_{\xi^{\prime}}^{\mathcal{C}^{\prime}} \rightarrow$ $\mathbf{M}^{\left(\xi, \mathcal{C}^{\prime}\right)}$, defined by $F(X, \mathcal{Q} \downarrow):=(X, \xi(\mathcal{Q} \downarrow))$ where $\mathcal{Q}$ again is a collection of $\mathcal{C}^{\prime}$ metrics and satisfies $\xi^{\prime}(\mathcal{Q} \downarrow)=\mathcal{Q} \downarrow$, i.e. $[\xi(\mathcal{Q} \downarrow)]^{\mathcal{C}^{\prime}} \downarrow=\mathcal{Q} \downarrow$. This last equality means that $G \circ F=1$, and from the remark above one sees that also $F \circ G=1$.

2. Let $(X, \mathcal{D})$ be any object in $\mathbf{M}_{\xi}^{\mathcal{C}}$. Consider $\left(X, \xi\left([\mathcal{D}]^{\mathcal{C}^{\prime}} \downarrow\right)\right)$; this clearly is an object of $\mathbf{M}^{\left(\xi, \mathcal{C}^{\prime}\right)}$ coarser than $(X, \mathcal{D})$. Let $f:(X, \mathcal{D}) \rightarrow(Y, \xi(\mathcal{Q} \downarrow))$ be a morphism where $\mathcal{Q}$ is a collection of $\mathcal{C}^{\prime}$-metrics. From $\xi(\mathcal{Q} \downarrow) \circ f \times f \subset \mathcal{D}$ it follows that $\mathcal{Q} \circ f \times f \subset[\mathcal{D}]^{\mathcal{C}^{\prime}}$. Consequently, from (E4),

$$
\xi(\mathcal{Q} \downarrow) \circ f \times f \subset \xi((\mathcal{Q} \downarrow \circ f \times f) \downarrow)=\xi((\mathcal{Q} \circ f \times f) \downarrow) \subset \xi\left([\mathcal{D}]^{\mathcal{C}^{\prime}} \downarrow\right),
$$

which proves that $f:\left(X, \xi\left([\mathcal{D}]^{\mathcal{C}^{\prime}} \downarrow\right)\right) \rightarrow(Y, \xi(\mathcal{Q} \downarrow))$ too is a morphism.

3 . This is nothing else but an application of 3.3

\section{Models for $\mathcal{C}$-Metrically generated CONStructs}

Given a base category $\mathcal{C}$ and $K: \mathcal{C} \rightarrow \mathcal{X}$ satisfying (I) and (D), we introduce two concrete functors which will play a crucial role in the sequel.

For any object $(X, \mathcal{D})$ in $\mathbf{M}^{\mathcal{C}}$, with basis of $\mathcal{C}$-metrics $\mathcal{P}$, we put

$$
F_{K}(X, \mathcal{D})=\sup _{p \in \mathcal{P}} K(X, p) .
$$

Note that this unambiguously defines $F_{K}: \mathbf{M}^{\mathcal{C}} \rightarrow \mathcal{X}$.

For any object $\underline{X}$ in $\mathcal{X}$ we put

$$
G_{K}(\underline{X})=(X, \mathcal{P} \downarrow)
$$

where $\mathcal{P}=\{p \in \mathcal{C}(X) \mid K(X, p) \leq \underline{X}\}$. Note that $\mathcal{P} \neq \emptyset$ in view of 2.3 . This defines $G_{K}: \mathcal{X} \rightarrow \mathbf{M}^{\mathcal{C}}$. From initiality properties it follows that $F_{K}$ is a functor, and from (I) it follows that $G_{K}$ is a functor. To avoid confusion, suprema in a topological construct $\mathcal{X}$ will, from now on, sometimes be denoted by $\sup ^{\mathcal{X}}$.

4.1. Theorem. A topological construct is $\mathcal{C}$-metrically generated if and only if it is concretely isomorphic to $\mathbf{M}_{\xi}^{\mathcal{C}}$ for some expander $\xi$ on $\mathbf{M}^{\mathcal{C}}$. 
Proof. Let $K: \mathcal{C} \rightarrow \mathcal{X}$ be a functor satisfying (I) and (D).

(1) By definition we have that $F_{K} \circ G_{K}(\underline{X}) \leq \underline{X}$ for any $\mathcal{X}$-object $\underline{X}$. To prove the other inequality, by (D), let $\mathcal{P}$ be a set of $\mathcal{C}$-metrics such that $\underline{X}=\sup _{p \in \mathcal{P}} K(X, p)$. If $G_{K}(\underline{X})=(X, \mathcal{D} \downarrow)$ with $\mathcal{D}$ as in the definition of $G_{K}$, then $\mathcal{P} \subset \mathcal{D}$ and thus $\underline{X} \leq \sup _{q \in \mathcal{D}} K(X, q)=F_{K}\left(G_{K}(\underline{X})\right)$. This proves that $F_{K} \circ G_{K}=1$.

(2) We now prove that $G_{K} \circ F_{K}$ defines an expander $\xi$ on $\mathbf{M}^{\mathcal{C}}$ (and therefore a concretely coreflective subconstruct $\mathbf{M}_{\xi}^{\mathcal{C}}$ of $\mathbf{M}^{\mathcal{C}}$ ) such that $\mathcal{X}$ is isomorphic with $\mathbf{M}_{\xi}^{\mathcal{C}}$.

For any $X$ and any meter $\mathcal{D}$ on $X$, let $\xi$ be defined by

$$
(X, \xi(\mathcal{D}))=G_{K}\left(F_{K}(X, \mathcal{D})\right) .
$$

That $G_{K} \circ F_{K} \geq 1$ is proved in the same way as in (1), and from this it follows that $\mathcal{D} \subset \xi(\mathcal{D})$, which shows that (E1) is fulfilled. To prove (E2), let $\mathcal{D}^{\prime} \subset \mathcal{D}$ be meters on $X$. Then noting that with regard to the action of $F_{K}$ it is always possible to choose bases $\mathcal{P}$ for $\mathcal{D}$ and $\mathcal{P}^{\prime}$ for $\mathcal{D}^{\prime}$ such that $\mathcal{P}^{\prime} \subset \mathcal{P}$, it follows at once from the definitions that $F_{K}\left(X, \mathcal{D}^{\prime}\right) \leq F_{K}(X, \mathcal{D})$ and that $G_{K}\left(F_{K}\left(X, \mathcal{D}^{\prime}\right)\right) \leq G_{K}\left(F_{K}(X, \mathcal{D})\right)$. That (E3) is fulfilled follows from

$$
G_{K} \circ F_{K} \circ G_{K} \circ F_{K}=G_{K} \circ 1 \circ F_{K}=G_{K} \circ F_{K} .
$$

Finally to prove that property (E4) is fulfilled, let $f: X \rightarrow X^{\prime}$ be any function, and let $\left(X^{\prime}, \mathcal{D}\right)$ be an object in $\mathbf{M}^{\mathcal{C}}$ with base $\mathcal{P}$. For any $p \in \mathcal{P}$ we have that $f:(X, p \circ f \times f) \rightarrow\left(X^{\prime}, p\right)$ is a morphism in $\mathcal{C}$, and hence we have that also $f: K(X, p \circ f \times f) \rightarrow K\left(X^{\prime}, p\right)$ is an $\mathcal{X}$-morphism, and with $\underline{X}^{\prime}=F_{K}\left(X^{\prime}, \mathcal{D}\right)$ and $\underline{X}=F_{K}(X, \mathcal{D} \circ f \times f)$ also $f: \underline{X} \rightarrow \underline{X}^{\prime}$ is an $\mathcal{X}$-morphism. Now, for $p \in \mathcal{P}$, $\bar{K}\left(X^{\prime}, p\right) \leq \underline{X}^{\prime}$ and so by (I) we know that $K(X, p \circ f \times f) \leq \underline{X}$. If $q \in \xi(\mathcal{D})$, then $K\left(X^{\prime}, q\right) \leq \underline{X^{\prime}}$, which by the foregoing observation implies that $K(X, q \circ f \times f) \leq \underline{X}$. This implies that $\xi(\mathcal{D}) \circ f \times f \subset \xi(\mathcal{D} \circ f \times f \downarrow)$.

Clearly, the restriction of $F_{K}$ to $\mathbf{M}_{\xi}^{\mathcal{C}}$ and $G_{K}$ are mutually inverse isomorphisms between $\mathbf{M}_{\xi}^{\mathcal{C}}$ and $\mathcal{X}$.

(3) Finally we prove that for any expander $\xi$ on $\mathbf{M}^{\mathcal{C}}, \mathbf{M}_{\xi}^{\mathcal{C}}$ is metrically generated. Define

$$
K_{\xi}: \mathcal{C} \longrightarrow \mathbf{M}_{\xi}^{\mathcal{C}}:(X, d) \mapsto(X, \xi(d \downarrow)) .
$$

If $f:(X, \mathcal{D}) \rightarrow\left(X^{\prime}, \mathcal{D}^{\prime}\right)$ is a morphism in $\mathbf{M}_{\xi}^{\mathcal{C}}$ and $\left(X^{\prime}, \xi(d \downarrow)\right) \leq\left(X^{\prime}, \mathcal{D}^{\prime}\right)$, then we have that $d \in \xi(d \downarrow) \subset \mathcal{D}^{\prime}$, and then also $d \circ f \times f \in \mathcal{D}$. Consequently we have that $\xi((d \circ f \times f) \downarrow) \subset \xi(\mathcal{D})=\mathcal{D}$. This proves that (I) is fulfilled. Next, let $(X, \mathcal{D})$ be an object in $\mathbf{M}_{\xi}^{\mathcal{C}}$ with basis $\mathcal{Q}$ of $\mathcal{C}$-metrics. Then we have that

$$
(X, \mathcal{D}) \leq \sup _{q \in \mathcal{Q}}^{\mathbf{M}^{\mathcal{C}}}(X, \xi(q \downarrow)) \leq \sup _{q \in \mathcal{Q}}^{\mathbf{M}_{\xi}^{\mathcal{C}}}(X, \xi(q \downarrow)) \leq(X, \mathcal{D}),
$$

where the second inequality follows from the fact that $\mathbf{M}_{\xi}^{\mathcal{C}}$ is concretely coreflective in $\mathbf{M}^{\mathcal{C}}$ as shown in 3.5

The expander in (2) of the foregoing proof is entirely determined by the functor $K$ and if necessary to avoid confusion it will be denoted $\xi_{K}$. Note that

$$
\xi_{K_{\xi}}=\xi \text { and that } K_{\xi_{K}}=G_{K} \circ K
$$

4.2. Proposition. Let $\mathcal{C}$ and $\mathcal{C}^{\prime}$ be base categories such that $\mathcal{C}^{\prime} \hookrightarrow \mathcal{C}$, and let $K$ : $\mathcal{C} \rightarrow \mathcal{X}$ be a functor satisfying (I) and (D). Let $\mathcal{Y}$ be the concretely reflective hull 
of $K\left(\mathcal{C}^{\prime}\right)$ in $\mathcal{X}$. Then the restriction of $K$ to $K^{\prime}: \mathcal{C}^{\prime} \rightarrow \mathcal{Y}$ also satisfies (I) and (D) and $\xi_{K^{\prime}}=\left(\xi_{K}\right)^{\prime}$.

Proof. That $K^{\prime}$ satisfies (I) and (D) follows from the fact that $\mathcal{Y}$ is concretely reflective in $\mathcal{X}$ and therefore initially closed in $\mathcal{X}$.

Now note that $\left.F_{K}\right|_{\mathbf{M}^{\mathcal{C}^{\prime}}}=F_{K^{\prime}}$. Indeed, let $(X, \mathcal{D})$ be an object in $\mathbf{M}^{\mathcal{C}^{\prime}}$ with basis $\mathcal{P}$ of $\mathcal{C}^{\prime}$-metrics. Then

$$
F_{K^{\prime}}(X, \mathcal{D})=\sup _{p \in \mathcal{P}}^{\mathcal{Y}} K^{\prime}(X, p)=\sup _{p \in \mathcal{P}}^{\mathcal{X}} K(X, p)=F_{K}(X, \mathcal{D}) .
$$

Further, for any object $(X, \mathcal{D})$ in $\mathbf{M}^{\mathcal{C}^{\prime}}$ we have that $e \in \xi_{K^{\prime}}(\mathcal{D})$ if and only if there exists a $\mathcal{C}^{\prime}$-metric $q$ such that $e \leq q$ and such that $K^{\prime}(X, q) \leq F_{K^{\prime}}(X, \mathcal{D})$, i.e. such that $K(X, q) \leq F_{K}(X, \mathcal{D})$ i.e. such that $q \in \xi_{K}(\mathcal{D})$. Thus $\xi_{K^{\prime}}(\mathcal{D})=\left(\xi_{K}\right)^{\prime}(\mathcal{D})$.

4.3. Proposition. Let $\mathcal{X}$ be metrically generated by $K: \mathcal{C} \rightarrow \mathcal{X}$. Then any concretely coreflective subconstruct $\mathcal{Y}$ of $\mathcal{X}$ is again metrically generated by the functor $C \circ K: \mathcal{C} \rightarrow \mathcal{Y}$ where $C$ is the $\mathcal{Y}$-coreflector.

Proof. This follows since $C$ preserves initial morphisms and suprema.

\section{EXAMPLES}

5.1. Pretopological, topological and completely regular spaces (1], 2], [5], [7]). Consider the following base categories: $\mathcal{C}=$ Met, $\mathcal{C}^{\prime}=$ "quasi-metric spaces" and $\mathcal{C}^{\prime \prime}=$ "metric spaces". For $d \in \mathcal{C}(X), \mathcal{T}_{d}$ is the pretopology on $X$ with neighborhood filter in $x \in X$ generated by $\left\{B_{d}(x, r) \mid r>0\right\}$ where $B_{d}(x, r)=\{y \mid d(x, y)<r\}$. This determines a concrete functor to the construct of pretopological spaces

$$
T: \mathcal{C} \longrightarrow \operatorname{PrTop}:(X, d) \mapsto\left(X, \mathcal{T}_{d}\right)
$$

together with its restrictions $T^{\prime}: \mathcal{C}^{\prime} \rightarrow$ Top and $T^{\prime \prime}: \mathcal{C}^{\prime \prime} \rightarrow$ Creg. To see that $f: T(X, p \circ f \times f) \rightarrow T(X, p)$ is initial in PrTop it is sufficient to observe that for $x \in X$ and $r>0: B_{p \circ f \times f}(x, r)=f^{-1} B_{p}(f(x), r)$. To see that $T(\mathcal{C})$ is initially dense in PrTop, let $\underline{X}$ be a pretopological space. For $A, V \subset X$ with $V$ a neighborhood of $A$ we put $p_{(A, V)}:=1_{A \times(X \backslash V)}$. Then

$$
\underline{X}=\sup _{V \text { nbhd of } A}\left(X, \mathcal{T}_{p_{(A, V)}}\right) .
$$

The expander $\xi_{T}$ associated with the functor $T$ is given in the following way (cfr. [4]): for any $\mathcal{D} \in \mathbf{M}(X), e \in \xi_{T}(\mathcal{D})$ if and only if

$$
\forall x \in X, \forall \epsilon>0, \exists d_{1}, \ldots, d_{n} \in \mathcal{D}, \exists \delta>0: \sup _{i=1}^{n} d_{i}(x, y)<\delta \Rightarrow e(x, y)<\epsilon .
$$

To deal with Top note that since all spaces $T(X, d)$ for $(X, d)$ an object in $\mathcal{C}^{\prime}$ are topological, the concretely reflective hull of $T\left(\mathcal{C}^{\prime}\right)$ is a subconstruct of Top. Conversely, every topological space $\underline{X}$ can be written as

$$
\underline{X}=\sup _{A \text { open }}\left(X, \mathcal{T}_{p_{(A, A)}}\right) .
$$

Now, since all these $p_{(A, A)}{ }^{\prime} s$ are quasi-metrics, Top is the concretely reflective hull of $T\left(\mathcal{C}^{\prime}\right)$. Applying 4.2 allows us to conclude that Top is metrically generated by $T^{\prime}: \mathcal{C}^{\prime} \rightarrow$ Top with associated expander the quasi-metric modification of $\xi_{T}$.

Analogously, Creg is metrically generated by $T^{\prime \prime}: \mathcal{C}^{\prime \prime} \rightarrow$ Creg with associated expander the metric modification of $\xi_{T}$. 
5.2. Quasi-uniform, uniform and proximity spaces ([1], [5], 7], [9, 11]). Consider the following base categories: $\mathcal{C}=$ "quasi-metric spaces", $\mathcal{C}^{\prime}=$ "metric spaces" and $\mathcal{C}^{\prime \prime}=$ "totally bounded metric spaces". For a quasi-metric $d, \mathcal{U}_{d}=$ stack $\left\{V_{d}^{\epsilon} \mid \epsilon>0\right\}$ where $V_{d}^{\epsilon}=\{(x, y) \mid d(x, y)<\epsilon\}$ is a quasi-uniformity. This assignment determines a concrete functor to the construct of quasi-uniform spaces,

$$
U: \mathcal{C} \rightarrow q \text { Unif }:(X, d) \mapsto\left(X, \mathcal{U}_{d}\right),
$$

together with its restrictions $U^{\prime}: \mathcal{C}^{\prime} \rightarrow$ Unif and $U^{\prime \prime}: \mathcal{C}^{\prime \prime} \rightarrow$ Prox to, respectively, the constructs of uniform spaces and of proximity spaces (which is isomorphic to the construct of totally bounded uniform spaces). It is well known that both Unif and $q$ Unif can be described by means of meters. For Unif we refer to the book of Gillman and Jerison [7], and for $q$ Unif we refer to the survey paper by Künzi [9]. It follows that $q \mathbf{U n i f}$ is isomorphic to $\mathbf{M}_{\xi_{U}}^{\mathcal{C}}$, where for any $\mathcal{C}$-meter $\mathcal{D}$ on $X$ we have $e \in \xi_{U}(\mathcal{D})$ (cfr. [4]), if and only if there exists a quasi-metric $r$ on $X$ such that $e \leq r$ and such that

$$
\forall \epsilon>0, \exists d_{1}, \ldots, d_{n} \in \mathcal{D}, \exists \delta>0: \sup _{i=1}^{n} d_{i}(x, y)<\delta \Rightarrow r(x, y)<\epsilon .
$$

Furthermore, Unif is concretely isomorphic to $\mathbf{M}_{\left(\xi_{U}\right)^{\prime}}^{\mathcal{C}^{\prime}}$ where $\left(\xi_{U}\right)^{\prime}$ is the metric modification of $\xi_{U}$. If we further restrict the functor $U$ to $\mathcal{C}^{\prime \prime}$, then $U\left(\mathcal{C}^{\prime \prime}\right)$ consists of all totally bounded metrizable uniform spaces. Hence the concretely reflective hull of $U\left(\mathcal{C}^{\prime \prime}\right)$ in Unif is a subconstruct of Prox. On the other hand, applying (D) for $U^{\prime}: \mathcal{C}^{\prime} \rightarrow$ Unif to a totally bounded uniform space $(X, \mathcal{U})$, then for some class of metrics $\mathcal{D}$ we have

$$
(X, \mathcal{U})=\sup _{d \in \mathcal{D}}^{\operatorname{Unif}}\left(X, \mathcal{U}_{d}\right)
$$

and since hence for all $d \in \mathcal{D},\left(X, \mathcal{U}_{d}\right)$ is totally bounded, it follows that $(X, \mathcal{U})$ is in the concretely reflective hull of $U\left(\mathcal{C}^{\prime \prime}\right)$.

Proposition 4.2 now implies that Prox is metrically generated by $U^{\prime \prime}: \mathcal{C}^{\prime \prime} \rightarrow$ Prox with expander $\left(\xi_{U}\right)^{\prime \prime}$, the totally bounded metric modification of $\xi_{U}$.

5.3. Preapproach, approach and uniform approach spaces ([3], [5], [10]). These categories were introduced by the authors in 3 and 10. Consider the base categories $\mathcal{C}=$ Met, $\mathcal{C}^{\prime}=$ "quasi-metric spaces" and $\mathcal{C}^{\prime \prime}=$ "metric spaces" and consider the functor to preapproach spaces

$$
A: \mathcal{C} \rightarrow \operatorname{PrAp}:(X, d) \mapsto(X, d \downarrow)
$$

together with its restrictions $A^{\prime}: \mathcal{C}^{\prime} \rightarrow \mathbf{A p}$ and $A^{\prime \prime}: \mathcal{C}^{\prime \prime} \rightarrow \mathbf{U A p}$ to, respectively, the constructs of approach spaces and of uniform approach spaces. Clearly $A$ preserves initial morphisms and $A(\mathcal{C})$ (resp. $A^{\prime}\left(\mathcal{C}^{\prime}\right)$ and $A^{\prime \prime}\left(\mathcal{C}^{\prime \prime}\right)$ ) are initially dense in $\operatorname{PrAp}$ (resp. Ap and UAp), 3] and [10]. The expander associated with $A$ is the following: for any $\mathcal{D} \in \mathbf{M}(X), e \in \xi_{A}(\mathcal{D})$ (cfr. [4]) if and only if

$$
\forall x \in X, \forall \epsilon>0, \forall \omega<\infty, \exists d_{1}, \ldots, d_{n} \in \mathcal{D}: e(x, \cdot) \wedge \omega \leq \sup _{i=1}^{n} d_{i}(x, \cdot)+\epsilon .
$$

Ap was shown in [10] to be the concretely reflective hull of $A\left(\mathcal{C}^{\prime}\right)$. Applying 4.2 allows us to conclude that $\mathbf{A p}$ is metrically generated by $A^{\prime}: \mathcal{C}^{\prime} \rightarrow \mathbf{A p}$ with associated expander the quasi-metric modification of $\xi_{A}$.

Analogously, UAp is metrically generated by $A^{\prime \prime}: \mathcal{C}^{\prime \prime} \rightarrow$ UAp with associated expander the metric modification of $\xi_{A}$. 
5.4. Bornological spaces generated by an $l^{\infty}$-structure ([6]). This important concretely reflective subconstruct of Bor was introduced by Frölicher and Kriegl in [6]. Its objects are those bornological spaces for which a set is bounded if and only if it contains no infinite countable subset, the only bounded subsets of which are the finite ones. Consider the base category $\mathcal{C}$ of all real-valued metric spaces and the following concrete functor:

$$
B: \mathcal{C} \rightarrow \text { Bor }^{\infty}:(X, d) \mapsto\left(X, \mathcal{B}_{d}\right),
$$

where $\mathcal{B}_{d}$ stands for the bornology generated by $d$, i.e., $A \in \mathcal{B}_{d}$ if and only if $\operatorname{diam}_{d}(A)<\infty$. It is easily seen that this is an $l^{\infty}$-bornology. Further, for $f$ : $(X, d \circ f \times f) \rightarrow\left(X^{\prime}, d\right)$ and $A \subset X, \operatorname{diam}_{d} f(A)=\operatorname{diam}_{d \circ f \times f} A$, which implies that $B$ preserves initial morphisms. $B(\mathcal{C})$ is initially dense since Bor $^{\infty}$ is the concretely reflective hull of $\left(\mathbb{R}, \mathcal{B}_{d_{E}}\right)$, where $d_{E}$ is the usual metric on $\mathbb{R}[6]$. Hence Bor ${ }^{\infty}$ is metrically generated by $B: \mathcal{C} \rightarrow$ Bor $^{\infty}$.

5.5. Reflexive relations (spatial graphs) and preordered sets (1, [5]). The construct Rere of reflexive relations (or spatial graphs [5]) is isomorphic to the coreflective subconstruct of PrTop consisting of finitely generated pretopological spaces, and thus by 4.3, is metrically generated. Rere is concretely isomorphic to $\mathbf{M}_{\xi}$ where

$$
\xi(\mathcal{D}):=\left\{e \text { quasi-pre-metric } \mid \sup _{d \in \mathcal{D}} d(x, y)=0 \Rightarrow e(x, y)=0\right\}
$$

for any meter $\mathcal{D}$. Likewise Prost, which is isomorphic to the coreflective subconstruct of Top consisting of finitely generated topological spaces, is metrically generated with expander the quasi-metric modification of $\xi$.

5.6. Measurable spaces ([12]). The construct Meas of measurable spaces is metrically generated. It suffices to consider the base category $\mathcal{C}$ of quasi-metric spaces and the functor

$$
S: \mathcal{C} \rightarrow \text { Meas }:(X, d) \mapsto\left(X, \sigma\left(\mathcal{T}_{d}\right)\right)
$$

where $\sigma\left(\mathcal{T}_{d}\right)$ stands for the Borel $\sigma$-algebra generated by the topology $\mathcal{T}_{d}$. Given $f:(X, d \circ f \times f) \rightarrow\left(X^{\prime}, d\right)$ it follows from the fact that $f^{-1}\left(\sigma\left(\mathcal{T}_{d}\right)\right)=\sigma\left(\mathcal{T}_{d \circ f \times f}\right)$ that $S$ preserves initial morphisms. If $(X, \mathcal{A})$ is a measurable space, then it is easily verified that

$$
(X, \mathcal{A})=\sup _{A \in \mathcal{A}}^{\mathrm{Meas}}\left(X, \sigma\left(\mathcal{T}_{p_{(A, A)}}\right)\right)
$$

Hence Meas is metrically generated by $S: \mathcal{C} \rightarrow$ Meas.

5.7. (Generalized) metric spaces (3, 9], [10]). Of course Met and any of its topological subconstructs $\mathcal{C}$ of quasi-metric spaces, pre-metric spaces or metric spaces, are all metrically generated. In all cases it suffices to take as base category $\mathcal{C}$ and the identity functor $\mathcal{C} \rightarrow \mathcal{C}$. The associated expander $\xi$ in all cases is given by $\xi(\mathcal{D})=\{\sup \mathcal{D}\} \downarrow$ on $\mathcal{C}$-meters.

\section{Counterexamples}

6.1. Bornological spaces (1], [6, [12]). In contrast with Bor $^{\infty}$, and in spite of the fact that every metric space gives rise to a natural associated bornology (see 5.4), the construct Bor of all bornological spaces itself is not metrically generated. Suppose that $K: \mathcal{C} \rightarrow$ Bor is a functor satisfying (I) and (D), mapping $(X, d)$ to $K(X, d)=\left(X, \mathcal{K}_{d}\right)$. Let $X$ be infinite, and let $\mathcal{F}$ be a non-principal ultrafilter on $X$. 
Then $\mathcal{B}_{\mathcal{F}}:=\{B \subset X \mid X \backslash B \in \mathcal{F}\}$ is a bornology on $X$. Consequently there exists a collection $\mathcal{D}$ of $\mathcal{C}$-metrics such that $\left(X, \mathcal{B}_{\mathcal{F}}\right)=\sup _{d \in \mathcal{D}}^{\text {Bor }} K(X, d)=\left(X, \bigcap_{d \in \mathcal{D}} \mathcal{K}_{d}\right)$. Since $\mathcal{B}_{\mathcal{F}} \neq \mathcal{P}(X)$, we may assume $\mathcal{K}_{d} \neq \mathcal{P}(X)$ for all $d \in \mathcal{D}$. From the fact that $\mathcal{F}$ is an ultrafilter it follows that if $A \notin \mathcal{B}_{\mathcal{F}}$, then $X \backslash A \in \mathcal{B}_{\mathcal{F}}$, and hence $X \backslash A \in \mathcal{K}_{d}$, hence $A \notin \mathcal{K}_{d}$ for all $d \in \mathcal{D}$. So we can conclude that $\mathcal{B}_{\mathcal{F}}=\mathcal{K}_{d}$ for all $d \in \mathcal{D}$, and hence $\left(X, \mathcal{B}_{\mathcal{F}}\right)=K(X, d)$ for all, and hence some, $d \in \mathcal{D}$. The number of bornologies of type $\mathcal{B}_{\mathcal{F}}$ for $\mathcal{F}$ an ultrafilter on $X$ is $2^{2^{|X|}}$; however, the number of $\mathcal{C}$-metrics on $X$ is dominated by $2^{|X|}$.

6.2. Pseudotopological and nearness spaces ([1, [2, [12, 8]). Although PrTop is metrically generated (see 5.1), going one step further and considering the construct PsTop of pseudotopological spaces also introduced by Choquet in 2 fails to produce a metrically generated construct. Take $\mathbb{N}$ as underlying set. Choosing any collection of non-principal ultrafilters on $\mathbb{N}$ and forcing them to converge to 0 as well as having all principal ultrafilters converge to their determining point fixes a pseudotopology. Therefore the number of pseudotopologies on $\mathbb{N}$ is at least $2^{2^{2^{\aleph_{0}}}}$. If PsTop were metrically generated, it would be concretely isomorphic to a subcategory of $\mathbf{M}$ by 4.1. However, the number of quasi-pre-metrics on $\mathbb{N}$ equals $2^{\aleph_{0}}$, and hence there are at most $2^{2^{\aleph_{0}}}$ meters on $\mathbb{N}$. An analogous technique allows us to show that also Near, the category of nearness spaces, is not metrically generated (cfr. 4]).

\section{REFERENCES}

[1] J. Adámek, H. Herrlich and G.E. Strecker. Abstract and Concrete Categories. John Wiley \& Sons, New York, 1990. MR1051419 (91h:18001)

[2] G. Choquet. Convergences. Ann. Univ. Grenoble, Sect. Math. Phys., 23 (1948), 57-112. MR0025716 (10,53d)

[3] E. Colebunders, R. Lowen. Topological quasitopos hulls of categories containing topological and metric objects, Cahiers Topol. Géom. Diff. Catég. 30 (1989), 213-228. MR 1029625 (91a:54015)

[4] E. Colebunders, R. Lowen and M. Sioen. Saturated collections of metrics, preprint.

[5] D. Dikranjan, W. Tholen. Categorical Structure of Closure Operators, Mathematics and its Applications, Kluwer Academic Publishers, 1995. MR1368854 (97i:18004)

[6] A. Frölicher and A. Kriegl. Linear Spaces and Differentiation Theory, Pure and Applied Mathematics, John Wiley \& Sons, 1988. MR0961256 (90h:46076)

[7] L. Gillman, M. Jerison. Rings of Continuous Functions, Springer Verlag, 1976. MR0407579 $(53: 11352)$

[8] H. Herrlich. A concept of nearness, Gen. Top. Appl., 4 (1974), 191-212. MR0350701 (50:3193)

[9] H.-P. Künzi. Nonsymmetric distances and their associated topologies: about the origins of basic ideas in the area of asymmetric topology, Handbook of the History of General Topology, Volume 3, Kluwer Academic Publishers, (2001), 853-968. MR1900267|(2003d:54001)

[10] R. Lowen. Approach Spaces: the Missing Link in the Topology-Uniformity-Metric Triad, Oxford Mathematical Monographs, Oxford University Press, 1997. MF 1472024 (98f:54002)

[11] S.A. Naimpally, B.D. Warrack. Proximity Spaces, Cambridge University Press, 1970. MR 0278261 (43:3992)

[12] G. Preuss. Theory of Topological Structures, Mathematics and its Applications, Kluwer Academic Publishers, 1988. MR0937052 (89m:54014)

Vrije Universiteit Brussel, Vakgroep Wiskunde, Pleinlaan 2, 1050 Brussel, Belgium E-mail address: evacoleb@vub.ac.be

Department of Mathematics and Computer Science, University of Antwerp, MiddelHEIMLAAN 1, 2020 ANTWERP, BElgium

E-mail address: bob.lowen@ua.ac.be 